\title{
Correction to: Male energy reserves, mate-searching activities, and reproductive success: alternative resource use strategies in a presumed capital breeder
}

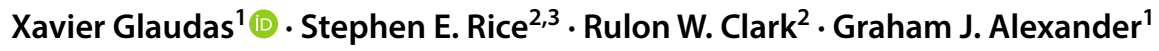

Published online: 13 October 2020

○) Springer-Verlag GmbH Germany, part of Springer Nature 2020

\section{Correction to: Oecologia \\ https://doi.org/10.1007/s00442-020-04755-0}

The authors would like to correct the error in table 4 which was incorrectly published in original version. Correct version of Table 4 is updated here.

The original article has been corrected.

The original article can be found online at https://doi.org/10.1007/ s00442-020-04755-0.

Xavier Glaudas

glaudasx@gmail.com

1 School of Animal, Plant and Environmental

Sciences, University of the Witwatersrand, P.O. Wits,

Johannesburg 2050, South Africa

2 Department of Biology, San Diego State University, San Diego, CA 92182, USA

3 Department of Natural Sciences, Fairmont State University, Fairmont, WV 26554, USA 
Table 4 Predictors (feeding group: control vs. fed; SVL $=$ snout-vent length) of mass change and behavioural frequencies in a South African population of adult male puff adders (Bitis arietans) during the mating season [linear mixed models with subject (ID) and year set as random factors; $n=28, \mathrm{ID}=20$, year $=3$ ]

\begin{tabular}{lllll}
\hline Model & $\beta$ & $P$ & $\Delta \mathrm{AIC}_{\mathrm{c}}$ & $R_{\mathrm{m}}^{2} / R_{\mathrm{c}}^{2}$
\end{tabular}

(a) Mass change (square root + constant $[=268]$; negative sign indicates larger change)
SVL+Feeding group*
$-0.24,-6.46(\mathrm{~F})$
$0.002,<0.001$
$0.45 / 0.67$
Feeding group*
$5.72(\mathrm{~F})$
$<0.001$
5.54
$0.30 / 0.64$
SVL
$-0.17$
0.10
15.85
$0.08 / 0.28$

(b) Ambush frequency (arcsine square root)

$\begin{array}{lllll}\text { SVL+Feeding group* } & -0.009,-0.13(\mathrm{~F}) & 0.004,0.009 & 0 & 0.24 / 0.69 \\ \text { SVL* } & -0.007 & 0.021 & 3.15 & 0.11 / 0.51 \\ \text { Feeding group } & -0.09(\mathrm{~F}) & 0.08 & 5.75 & 0.07 / 0.48\end{array}$

(c) Resting frequency (arcsine square root)

$\begin{array}{lllll}\text { SVL+Feeding group* } & 0.008,0.09(\mathrm{~F}) & 0.003,0.02 & 0 & 0.23 / 0.74 \\ \text { SVL }^{*} & 0.007 & 0.016 & 2.05 & 0.13 / 0.59 \\ \text { Feeding group } & 0.07(\mathrm{~F}) & 0.14 & 5.8 & 0.05 / 0.53\end{array}$

(d) Moving frequency (arcsine square root)

\begin{tabular}{lllll} 
Feeding group & $-0.01(\mathrm{~F})$ & 0.74 & 2.88 & $0.002 / 0.38$ \\
SVL & -0.0006 & 0.82 & 2.94 & $0.001 / 0.38$ \\
SVL + Feeding group & $-0.0009,-0.02(\mathrm{~F})$ & $0.77,0.71$ & 6.07 & $0.005 / 0.38$ \\
\hline
\end{tabular}

Models are presented in order of preference based on $\mathrm{AIC}_{\mathrm{c}}$ scores. F refers to the fed group, and $R_{\mathrm{m}}^{2}$ and $R_{\mathrm{c}}^{2}$ are the marginal and conditional $R^{2}$ values, respectively. The asterisks indicate the models that are statistically better than the null model. In case the first listed model has $\Delta \mathrm{AIC}_{\mathrm{c}} \neq 0$, the top model is the null model 